OPEN ACCESS

Edited by:

Maurice Chacron,

McGill University, Canada

Reviewed by:

Leonard Maler,

University of Ottawa, Canada

Harold H. Zakon,

University of Texas at Austin,

United States

*Correspondence:

Gary Marsat

gary.marsat@mail.wvu.edu

Received: 28 March 2019

Accepted: 20 May 2019

Published: 04 June 2019

Citation:

Motipally SI, Allen KM, Williamson DK and Marsat G (2019) Differences in

Sodium Channel Densities in the Apical Dendrites of Pyramidal Cells of the Electrosensory Lateral Line Lobe. Front. Neural Circuits 13:41. doi: 10.3389/fncir.2019.00041

\section{Differences in Sodium Channel Densities in the Apical Dendrites of Pyramidal Cells of the Electrosensory Lateral Line Lobe}

\author{
Sree I. Motipally, Kathryne M. Allen, Daniel K. Williamson and Gary Marsat* \\ Department of Biology, West Virginia University, Morgantown, WV, United States
}

Heterogeneity of neural properties within a given neural class is ubiquitous in the nervous system and permits different sub-classes of neurons to specialize for specific purposes. This principle has been thoroughly investigated in the hindbrain of the weakly electric fish $A$. leptorhynchus in the primary electrosensory area, the Electrosensory Lateral Line lobe (ELL). The pyramidal cells (PCs) that receive inputs from tuberous electroreceptors are organized in three maps in distinct segments of the ELL. The properties of these cells vary greatly across maps due to differences in connectivity, receptor expression, and ion channel composition. These cells are a seminal example of bursting neurons and their bursting dynamic relies on the presence of voltage-gated $\mathrm{Na}^{+}$channels in the extensive apical dendrites of the superficial PCs. Other ion channels can affect burst generation and their expression varies across ELL neurons and segments. For example, SK channels cause hyperpolarizing after-potentials decreasing the likelihood of bursting, yet bursting propensity is similar across segments. We question whether the depolarizing mechanism that generates the bursts presents quantitative differences across segments that could counterbalance other differences having the opposite effect. Although their presence and role are established, the distribution and density of the apical dendrites' $\mathrm{Na}^{+}$channels have not been quantified and compared across ELL maps. Therefore, we test the hypothesis that $\mathrm{Na}^{+}$channel density varies across segment by quantifying their distribution in the apical dendrites of immunolabeled ELL sections. We found the $\mathrm{Na}^{+}$channels to be two-fold denser in the lateral segment (LS) than in the centro-medial segment (CMS), the centro-lateral segment (CLS) being intermediate. Our results imply that this differential expression of voltage-gated $\mathrm{Na}^{+}$ channels could counterbalance or interact with other aspects of neuronal physiology that vary across segments (e.g., SK channels). We argue that burst coding of sensory signals, and the way the network regulates bursting, should be influenced by these variations in $\mathrm{Na}^{+}$channel density.

Keywords: sodium channels, bursting, weakly electric fish, feature detection, apical dendrites, backpropagation 


\section{INTRODUCTION}

Neurons possess a variety of ion channels and membrane proteins that shape their response properties, from the classical $\mathrm{Na}^{+}$and $\mathrm{K}^{+}$ion channels generating action potentials to G-protein coupled receptors (e.g., Prešern et al., 2015; Duménieu et al., 2017; Lizbinski et al., 2018). Heterogeneity in neuronal physiology can be understood through two complementary principles. One perspective stresses that a given neural output can result from various composition of channels and receptors. This principle was most obviously demonstrated in the stomatogastric ganglion of crabs, where an identical motor output pattern could be generated using networks which neurons differed widely in their channel composition (Prinz et al., 2004). This work highlighted that a change in one element of the neuron's physiology can be compensated by changes in another element.

The complementary, but non-exclusive, principle is also a basic concept in neuroscience. This principle argues that changes in membrane proteins (e.g., voltage gated ions channels) are necessary for specialization of neurons, and result in different neural outputs (Hille, 2001). An example of this principle, central to the subject of our study, comes from neurons that possess specific ionic conductances responsible for generating burst firing (Krahe and Gabbiani, 2004). The neuron's bursting dynamic could not be possible without these specific ion channels and thus their role in neural coding is changed by this bursting dynamic.

Bursting is observed in various sensory systems and typically fulfills the same function: signaling the occurrence of specific spatio-temporal patterns of inputs (Gabbiani et al., 1996; Kepecs et al., 2002). In the visual system, bursts signal edges and sharp contrasts (Lesica and Stanley, 2004), in the cricket auditory system they signal salient ultrasound pulses typical of insectivorous bats (Marsat and Pollack, 2006, 2012) and in the electrosensory system they signal prey-like peaks in signal amplitude or communication signals (Gabbiani et al., 1996; Oswald et al., 2004; Marsat et al., 2009). The presence of a bursting mechanism is key in shaping the neuron's role in the sensory pathway. The present study focuses on this bursting property and investigates variations in the ion channels responsible for burst generation in the sensory system of weakly electric fish.

The electrosensory lateral line lobe (ELL) is the primary sensory area in the hindbrain of gymnotid weakly electric fish and the main output neurons, pyramidal cells (PCs), possess a well characterized bursting mechanism (Turner et al., 1994; Doiron et al., 2002). PCs, particularly the more superficial ones, have extensive apical dendrites dedicated to receiving feedback inputs, but these dendrites also support the generation of bursts. These apical dendrites extend several $100 \mu \mathrm{m}$ into the molecular layer (Figure 1A) dorsal to the PC layer (PCL; Bastian and Courtright, 1991). They contain TTX-sensitive voltage-gated sodium channels (Nav channels). When an action potential is initiated in the cell, the somatic potential backpropagates actively $\sim 200 \mu \mathrm{m}$ up the apical dendrites due to the Nav conductance. Current from the backpropagating action potential then flows back down to the soma passively causing a depolarizing
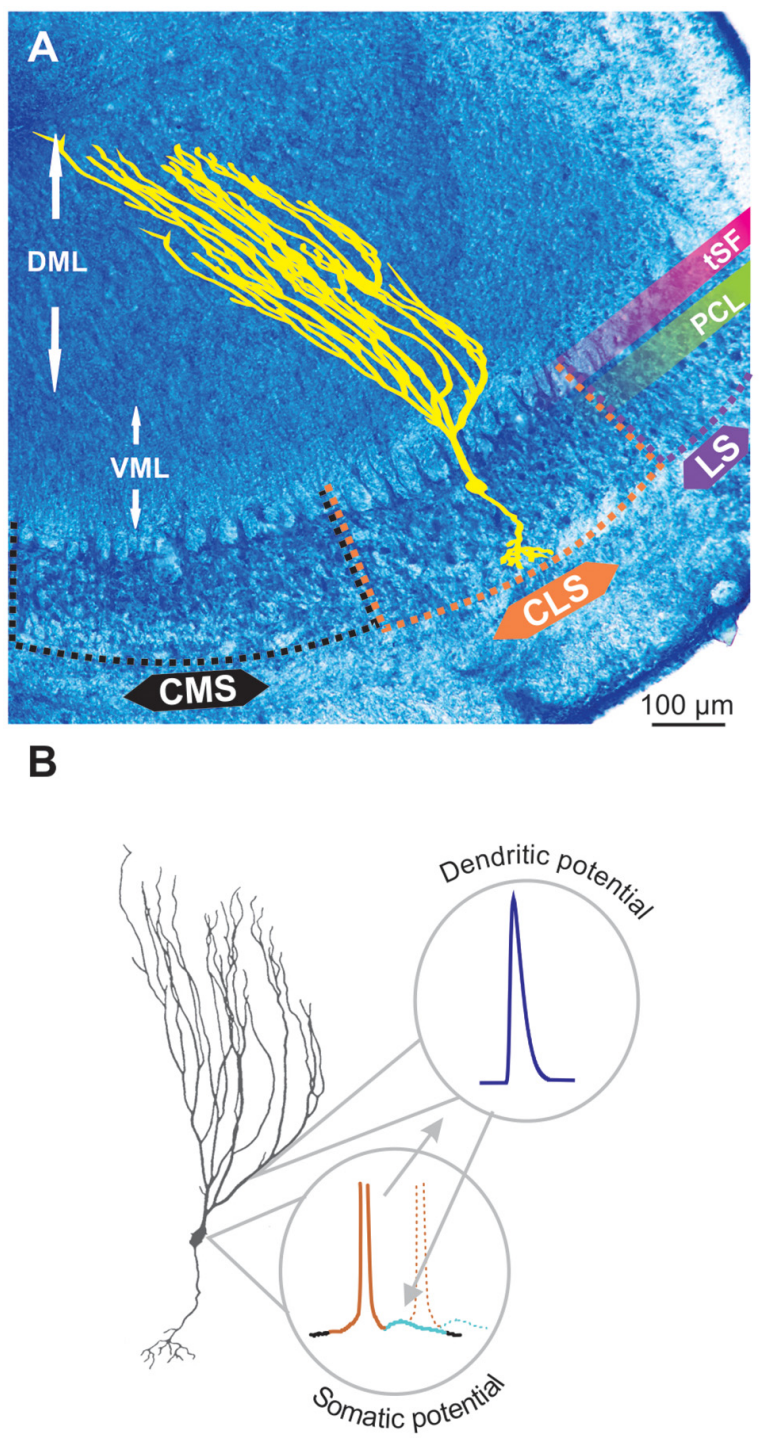

FIGURE 1 | Pyramidal cell (PC) and backpropagation. (A) Transverse section through the electrosensory lateral line lobe (ELL), the primary sensory area located in the hindbrain of gymnotid weakly electric fish. The structure is layered and the main output neurons-PCs (yellow schematic) are organized in several topographic maps: medial, centro-medial, centro-lateral and lateral segments (MS, CMS, CLS and LS, respectively). The latter three segments receive inputs from tuberous electroreceptors. The $\mathrm{PC}$ layer $(\mathrm{PCL})$ contains the soma of these neurons whereas the ventral and dorsal molecular layers (VML and DML, respectively) contain the extensive apical dendrites of the PCs (illustrated in yellow). The tractus stratum fibrosum (tSF) is an easily identifiable band (see also Figure 2) separating the PCL from DML. (B) Backpropagation and bursting. The ON PCs possess a small basilar dendritic bush receiving feedforward inputs, extensive apical dendrites (particularly long in superficial PCs) receiving feedback and an axon (not depicted here). Apical dendrites contain Nav channels such that, when an action potential is elicited in the soma (brown portion of the voltage schematic), it propagates up and back down the apical dendrites causing a depolarizing after potential (DAP; light blue) at the soma. The DAP increases the probability that subsequent action potentials (e.g., dashed line) are fired immediately after the first one thus leading to bursting.

after-potential (DAP) after each spike (Figure 1B; Turner et al., 1994). In slices and in models, this backpropagation mechanism 
can trigger a sequence of several spikes with increasingly shorter interspike intervals (ISIs). This stereotyped bursting dynamic, named ghostbursting (Doiron et al., 2002), might not unfold in the same way in vivo, where bursts are typically truncated to be only a few spikes-long. Nevertheless, backpropagation and the DAP are an integral part of the bursting dynamic and enable burst-coding of sensory signals (Oswald et al., 2007).

Response properties of ELL PCs are shaped by a variety of additional factors that vary across cell subtypes (Maler, 2007; Chacron et al., 2011). PCs are classified based on their location in the ELL layer. Superficial and intermediate PCs have extensive apical dendrites while deep PCs have short apical dendrites and fulfil a different role in the circuit (Bastian and Nguyenkim, 2001; Bastian et al., 2004). PCs receive inputs from receptors either directly or through an inverting interneuron. This leads to response patterns typical of ON-cells and OFF-cells, respectively (Maler, 1979; Clarke et al., 2015). Both types have the same burst dynamic. The ELL is organized in several topographic maps. While the map of the medial segment is driven by a separate category of passive ampullary electroreceptors, the centro-medial, centro-lateral and lateral segments (CMS, CLS and LS, respectively) receive inputs from the tuberous receptors sensitive to the fish's self-generated electric signal (Kawasaki, 2005). These areas each play a critical role in prey detection, communication, and navigation. In this study, we focus on those three ELL maps responsible for processing this activelygenerated electrosensory signal.

PCs from the three maps vary widely in their response properties. These differences are due to variations in network connectivity, ion channels composition, expression of neuromodulator receptors and more (Ellis et al., 2008; Maler, 2009; Krahe and Maler, 2014). These differences in properties allow the specialization of the three maps for different purposes: whereas the CMS is well suited for the localization of small near-by objects such as prey, the more lateral segments (CLS and especially LS) might specialize for processing communication signals (Metzner and Juranek, 1997; Marsat et al., 2009). Despite these differences in properties and function, all segments display similar bursting rates (Krahe et al., 2008). Although some small differences in burst patterns have been noted (Turner et al., 1996; Metzner et al., 1998; Mehaffey et al., 2008), it is unclear to what extent burst dynamics varies across segments since no fundamental differences have been documented in burst coding (Metzner et al., 1998; Oswald et al., 2004; Krahe et al., 2008). Large differences, particularly in bursting rates, could have been expected across different segments given the known differences in conductances affecting bursting. SK channels, which generate a hyperpolarizing after-potential, vary in expression across PCs subtypes and segments. They are particularly prevalent in the LS where they oppose the DAP-based burst generation mechanism (Ellis et al., 2007a, 2008). Serotonin receptors are also expressed differently, with LS cells expressing more, and when activated serotonergic inputs can enhance bursting propensity (Johnston et al., 1990; Deemyad et al., 2011, 2013). It is clear that many factors interact to shape bursting and it is possible that similarities in burst coding across PC subtypes and segments happens despite differences in intrinsic configurations, rather than because they have identical physiology.

Variation in one element central to the burst-generation mechanism has not been examined yet: Nav channel expression in the apical dendrites of PCs. Considering the differences related to the burst mechanism noted above, we hypothesize that variations in the expression of Nav channels will also be observed across segments. To test this hypothesis, we performed immunocytochemistry on ELL slice labeling Nav channels in the molecular layer of the three segments of the ELL. We observed Nav expression throughout the molecular layer and we show that it is denser in LS than CMS. We argue that this differential expression should have functional consequences on response properties but that it is hard to determine how it interacts with the many other differences seen across segments.

\section{MATERIALS AND METHODS}

\section{Brain Slices Preparation}

Apteronotus leptorhynchus fish used for experiments were wild-caught and purchased from a tropical fish supplier. They were maintained in home tanks $(61 \times 30.5 \times 50.8 \mathrm{~cm})$ at $26-27^{\circ} \mathrm{C}, 250-300 \mu \mathrm{S}$ on inverted light cycles, fed ad libitum and were provided with environmental enrichment. Fish of either sex were anesthetized in tank water with MS-222 (3-amino benzoic acid ethyl ester, Western Chemicals Inc.) and respirated with oxygen bubbled MS-222 water during perfusion. All chemicals were obtained from Fisher Scientific (Hampton, NH, USA) unless otherwise noted. Heart was surgically exposed and intracardial perfusion was performed via the Conus arteriosus with $5 \mathrm{ml}$ of cold $0.9 \%$ saline containing Heparin (\#9041-08-1), $\mathrm{NaNO}_{2}$ (\#S25560) and $\mathrm{NaCl}$ (\#7647-14-5) which is followed by perfusion with $40 \mathrm{~mL}$ of cold 4\% paraformaldehyde (PFA; Electron Microscopy Sciences, \#RT-15714) in 1×-phosphate buffered saline (PBS), pH-7.3. Whole brains were surgically removed and post-fixed in $4 \%$ PFA in $1 \times$ PBS for $4 \mathrm{~h}$ at $4^{\circ} \mathrm{C}$ and were washed three times for $15 \mathrm{~min}$ each in $1 \times \mathrm{PBS}$ at $4^{\circ} \mathrm{C}$. Brains were sequentially cryoprotected in $20 \%$ and $30 \%$ sucrose (\#S25590) in $1 \times-P B S$, $\mathrm{pH}-7.3$ until they were completely saturated and later incubated in 1:1 mixture of $30 \%$ sucrose solution and optical cutting temperature (OCT) compound (Electron Microscopy Sciences, \#62550-01) for 1-2 h before embedding in OCT. Dry-ice chilled $100 \%$ ethanol was used to freeze the brain in OCT in a cryomold and the mold was incubated at $-80^{\circ} \mathrm{C}$ for $1-2 \mathrm{~h}$ before sectioning. 15-20 $\mu \mathrm{m}$ thick true-transverse brain sections were obtained using cryostat (Leica 1850) and the slides were stored at $4^{\circ} \mathrm{C}$ for immediate processing or stored at $-20^{\circ} \mathrm{C}$ until use.

\section{Immunohistochemistry}

Brain sections were immunoreacted for Nav in situ through the following procedure. Sections were washed three times with $1 \times$-PBS, pH-7.3 for 5 min each and were blocked for $1 \mathrm{~h}$ in $5 \%$ normal goat serum (\#005-000-121, Jackson Immuno Research) in PBSAT $(1 \times$ PBS, $5 \mathrm{mM}$ Sodium Azide and 0.1\% Triton X-100). Blocking was followed by 1 -h incubation with Anti-Pan Nav 
(Alomone labs, \#ASC003; 1:50) and purified Mouse Anti-MAP II (BD Biosciences, \#556320; 1:400) primary antibodies in blocking buffer at room temperature. The Anti-Pan Nav antibody was raised in rabbits and was shown to selectively bind to the $\mathrm{Na}$ channels in apteronitids' electric organ with a protein size of $250-260 \mathrm{kDa}$ (Ban et al., 2015). Later, brain sections were transferred to $4^{\circ} \mathrm{C}$ for overnight incubation. Note that the MAP2 antibody used in the current study only stains the high molecular weight isoforms of MAP2 and does not recognize low molecular weight MAP2 isoforms or other microtubule proteins. In addition, MAP2 is mainly concentrated in the dendritic part of the nerve cells (Olesen, 1994), this might possibly explain the comparatively fainter MAP2 labeling observed in the cell bodies.

Sections were washed four times with $1 \times-$ PBST $(1 \times$ PBS and $0.1 \%$ Triton X-100), pH-7.3 for 15 min each and were incubated with Goat anti-rabbit Alexa 488 (Life Technologies, \#A-11008; 1:500) and Goat anti Mouse Alexa 546 (Thermofisher, \#A-11030; 1:500) secondary antibodies for $3 \mathrm{~h}$ at room temperature in an enclosed moist chamber. Sections were washed four times with $1 \times$-PBS, pH-7.3 for 15 min each and were mounted in Vectashield (Vector Laboratories, \#H-1000) and coverslipped. Selectivity of the labeling was confirmed with several controls: an absorption control where the Nav antibody is incubated with the immunogen (Supplementary Figure S1), a control with no primary antibody (Supplementary Figure S2) and a quantification of Nav labeling in regions where no Nav channels are expected to be found. For this last control, we selected 27 different $10 \times 10 \mu \mathrm{m}$ areas in the gaps between dendrite in the tractus stratum fibrosum (tSF) layer (where we do not expect Nav channels to be present; see Figure 2D) and compared the Nav labeling to 27 areas selected randomly on the same scans but in the middle of the dendritic arbors in the molecular layer. As expected, minimal staining was found (gaps in tSF: 0.009 puncta/ $/ \mathrm{m}^{2}$; molecular layer: 0.1 puncta/ $/ \mathrm{m}^{2} ; T$-Test: $p<10^{-6}$ ) proving that puncta of labeling are not simply an artifact and randomly distributed on the tissue.

\section{Western Blot}

The specificity of polyclonal Anti Pan-Nav antibody was evaluated using western blot analysis (Figure 3F). Unperfused whole brain was surgically removed and freezed in liquid nitrogen and immediately homogenized with cold homogenization buffer containing $250 \mathrm{mM}$ Sucrose, $1 \mathrm{mM}$ EDTA, $10 \mathrm{mM}$ Tris HCL and protease inhibitor cocktail (\#4693132001, Sigma Aldrich), pH-7.2, on pre-chilled mortar and pestle. Tissue sample was sonicated using two $10 \mathrm{~s}$ pulses with 30 -s interval between each sonication. Sample was centrifuged at $500 \times g$ for $10 \mathrm{~min}$ to remove intact cells, nuclei and cell debris, and the supernatant was centrifuged at $100,000 \times g$ for $1 \mathrm{~h}$ at $4^{\circ} \mathrm{C}$. Supernatant was discarded, and the pellet was resuspended in homogenization buffer and centrifuged at $100,000 \times$ at $4^{\circ} \mathrm{C}$ for $1 \mathrm{~h}$. Resultant supernatant was discarded and the pellet containing the membrane fractions was used to run western blot.

For western blot, $1 \times$ LDS (\#NP0008, Thermofisher) was added to the protein samples which was then heated for $10 \mathrm{~min}$ at $70^{\circ} \mathrm{C}$. Five microliter of the sample was loaded into
4\%-6\% polyacrylamide gel along with Precision Plus Protein ${ }^{\mathrm{TM}}$ Dual Color Standard (\#1610374S, Bio-Rad) and was run at $120 \mathrm{~V}$. Transfer was done at $100 \mathrm{~mA}$ for $22 \mathrm{~h}$ at $4^{\circ} \mathrm{C}$ and the nitrocellulose membrane (\#162-0094, Bio-Rad) was blocked for $1 \mathrm{~h}$ with $5 \%$ BSA and $0.05 \% \mathrm{NaN} 3$ in $1 \times$ TBST ( $1 \times$ TBS, $0.1 \%$ Tween) under agitation. Primary antibody diluted in the blocking buffer (1:200) was applied to the membrane and incubated overnight at $4{ }^{\circ} \mathrm{C}$ under gentle agitation. After incubation, membrane was washed three times with $1 \times$ TBST for 15 min each. HRP conjugated anti-rabbit secondary antibody (\#A0545, Sigma) was applied at 1:10,000 dilution and agitated for $1 \mathrm{~h}$ at room temperature, which was followed by three 15-min washes with $1 \times$ TBST. ECL substrate (\#RPN2109, GE Healthcare) was added and the membrane was imaged on FluorChemQ system (Protein Simple).

\section{Nav Puncta Density Quantification}

Scans were obtained on FV-1000 Fluoview confocal microscope and minor brightness adjustments were made using Fluoview software. All of the scans were imaged using $60 \times$ oil immersion lens at $2 \times$ digital zoom unless otherwise noted. Scan size $\mathrm{X}^{*} \mathrm{Y}$ is set at $105.4^{*} 105.4 \mu \mathrm{m}$ with $\mathrm{Z}$ at $0.5 \mu \mathrm{m}$. Differences in the spatial distribution across the brain maps are assessed by quantitative image analysis using VAA-3D software. ImageJ software was used to perform image normalization. To measure the spatial distribution of dendritic Nav channels across different ELL maps (LS, CLS and CMS), the dorsal edge of the tSF layer is chosen as reference location $(0 \mu \mathrm{m})$. Scans covered the first $600-800 \mu \mathrm{m}$ of apical dendrites as they project dorsally through the ventral molecular layer (VML) and dorsal molecular layer (DML) layers.

In each scan, 2-4 portions of dendrites with clear MAP2 labeling were chosen prior to looking at the Nav labeling to prevent biased choice of dendrite based on expected channel density. Nav labeling was quantified by manually marking each punctum visually identified on the chosen dendrites. $\mathrm{XY}$ coordinates of each punctum and dendrites were stored for analysis. We were conservative in assigning a punctum as belonging to a dendrite and the numbers presented in this article should be viewed as a lower-bound estimate. Note that any bias in quantifying puncta within a segment is also inherent to the quantifications done across segments, and therefore, the differences observed in Nav density across the segments are unlikely to be affected by such biases.

To standardize the identification of the edge of the tSF layer (defined as location $0 \mu \mathrm{m}$ ) a Matlab (Mathworks, Natick, MA, USA) program was created to plot the density profile of the gaps present in the tSF layer along with the pixel intensity profile of the scan (see Figure 2D and explanation in the legend).

A series of slightly overlapping scans of the entire extent of each map were obtained (Figure 2A) and the bleached area from the overlapped portion as well as a local landmark was used to obtain the start point $\mathrm{x}$-coordinate of each subsequent scan of the map. This allowed us to determine the orthogonal distance of each scan, dendrite and punctum from the tSF edge. Since dendrites do not travel orthogonally to the tSF edge and the scan orientation (Figures 2B,C), we had to correct the distances using dendrites' angles. To do so, in each scan we 

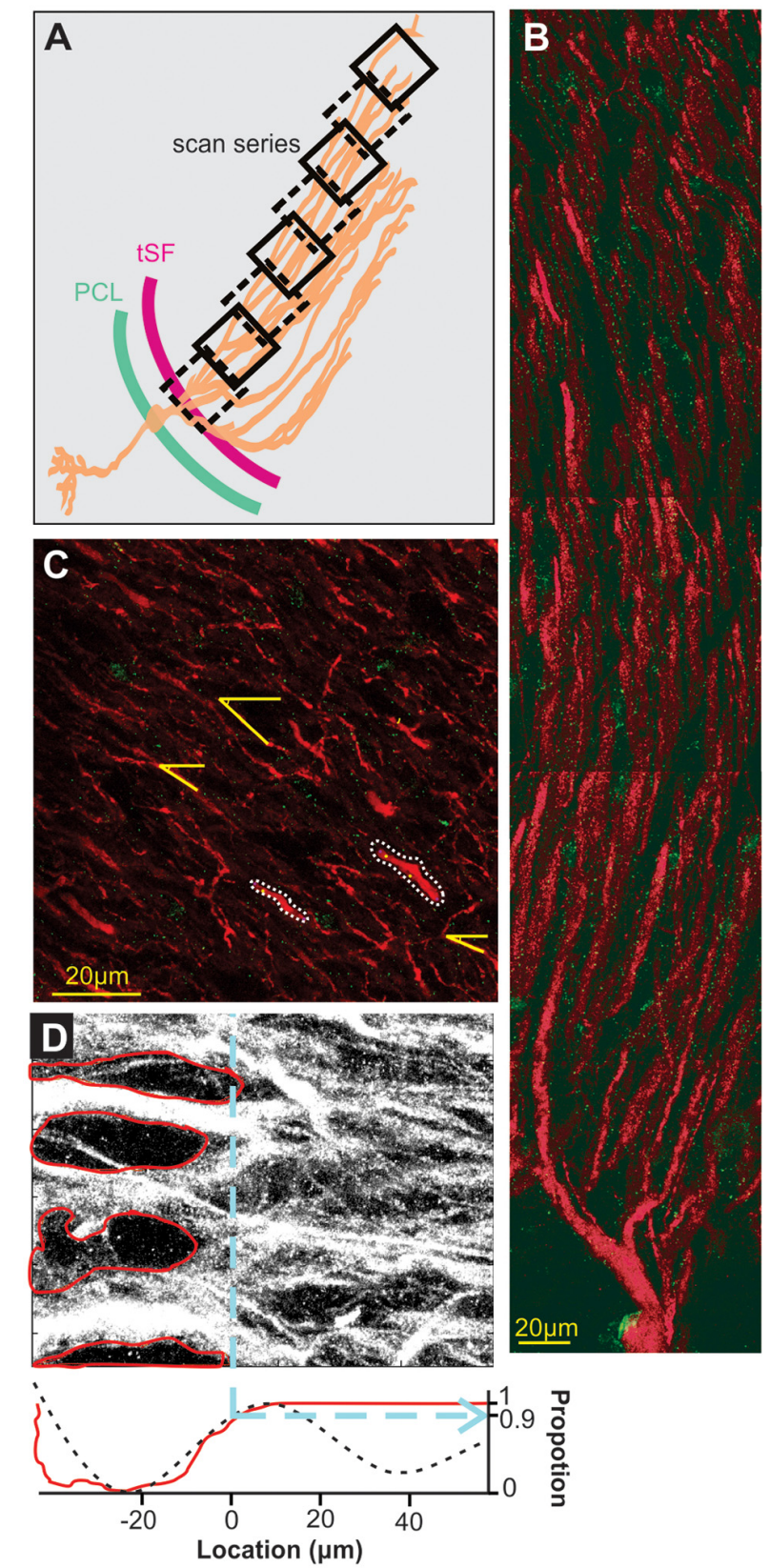

FIGURE 2 | Scanning and localization procedure. (A) Scans arrangement. Each $105 \times 105 \mu \mathrm{m}$ scan is positioned relative to the transverse section of the ELL so that the first one of a series contains the tSF layer and subsequent ones overlap the previous and follow the orientation of dendrites in the molecular layer. Eight successive scans or more were performed to cover at least $600 \mu \mathrm{m}$ of apical dendrites. (B) A series of scans showing the extensive apical dendritic bush in the molecular layer. Although we did not attempt to reconstruct and isolate single $\mathrm{PC}$, our successive scans follow the apical dendrites of PCs. Red MAP2 labeling marks the inside of the dendrites and green labeling highlights the position of Nav channels. Since no distinction could be made between ON and OFF cells based on the immunolabeling used, all the dendrites quantified in the study are mixed, either ON or OFF-cells, and possibly VML interneurons (although it is unknown if they express dendritic Nav). (C) Dendrite selection and angle. Based solely on the MAP2 labeling (Nav labeling not displayed) we selected several clearly defined

(Continued)
FIGURE 2 | Continued

dendrites per scans for quantification (e.g., outlined with white dashes). Since dendrite orientation relative to the scan orientation is not orthogonal, we measured, in each scan, the average relative orientation of dendrites based on several dendrites per scan (yellow lines). This allowed us, using the position on the $x$-axis of the scan, the angle of dendrites and trigonometry, to determine a more accurate position of dendrites along the dendritic bush. (D) Determination of tSF dorsal edge to set it as location " $0 \mu \mathrm{m}$ ". The stratum fibrosum tract is characterized by large circular areas without MAP2 labeling between the large proximal apical dendrite shafts. We determined the dorsal edge of this layer by first highlighting the visible holes in labeling (red in the top image) and constructing a pixel histogram (red curve, bottom plot) along the $x$-axis based on the pixels outside these areas. Second, we used the raw pixel intensity values to build a histogram along the $x$-axis and smoothed it by fitting a triple sinusoid function (black dashes, bottom plot). Both histograms were normalized between 0 and 1 and we took the 0.9 mark of the rising slope to determine the $0 \mu \mathrm{m}$ location (dashed blue line). We confirmed both histograms gave similar results and used the average of the two calculated values to set the 0-mark.

selected randomly five clear portions of dendrites and measured their angle relative to the scan's frame (Figure 2C). Using the average angle of the selected dendrites in each scan and trigonometry, orthogonal distances could be converted into the estimated distance (i.e., distances that take into account the general curvature of dendrites). This estimate is still an underestimation of actual distance along the dendritic tree since dendrites are often not completely straight over one scan, and also any z-plane curvature was ignored. Note that we sectioned the hindbrain in a true-transverse orientation minimizing the curvature of dendrites in the z-plane over the proximal apical dendrites. This underestimation does not affect our conclusions but should be noted. Data analysis was performed with Matlab (Mathworks, Naticks, MA, USA) and statistics with JMP (SAS Institute Inc., Cary, NC, USA).

\section{Burst ISI Characterization}

Fish were prepared for in vivo electrophysiological recordings as described in Marsat et al. (2009) where recording and analysis methods are also described in detail. Briefly, superficial $\mathrm{ON}$ and OFF-cells of the three segments were targeted and spontaneous activity was recorded for $60 \mathrm{~s}$. Recorded spike trains were binarized and burst identified by first constructing an ISI histogram of the entire spike train and identifying the upper interval limit characteristic of the cell's burst ISIs (for more details see Marsat and Pollack, 2012). Identified bursts were then used to calculate their ISI distribution. All procedures on animals were authorized by West Virginia University's IACUC (protocol \#13-0103).

\section{RESULTS}

To study the distribution of Nav channels along the apical dendrites of PCs we used a pan-Nav antibody known to bind selectively to these channels in other tissues of this species (Ban et al., 2015). We confirmed the selectivity of the antibody in brain tissue by performing a Western blot (Figure 3F). To identify the position of the labeled channels relative to the dendrites, we also used a MAP2 antibody that densely labels the 

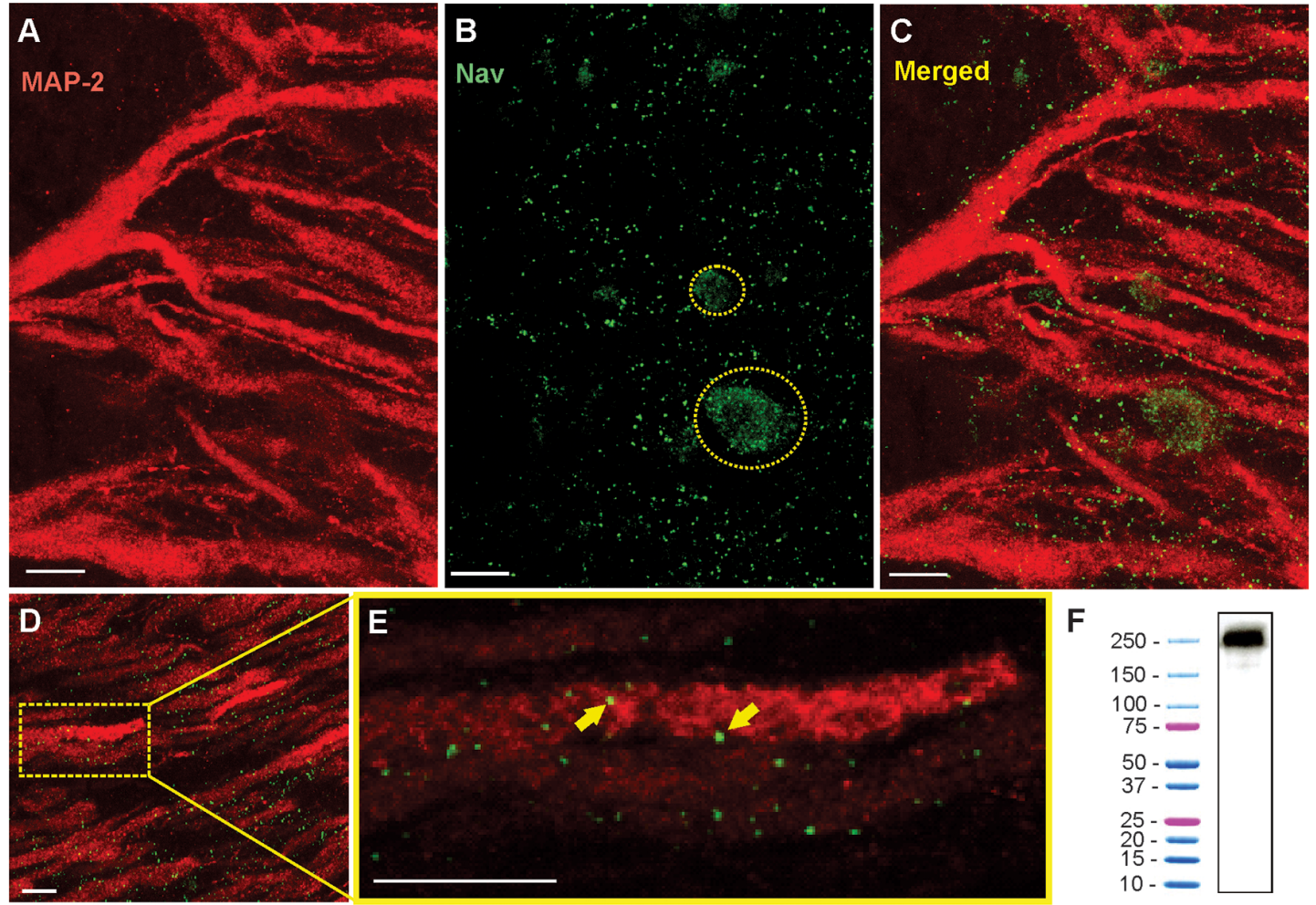

FIGURE 3 | Immunohistochemistry shows the punctate expression of Nav channels on the apical dendrites. (A) Confocal image of a $16 \mu \mathrm{m}$ section of ELL showing the red Anti-MAP2 labeled apical dendrites projecting through tSF and VML. White scale bars are $10 \mu \mathrm{m}$ in all panels. (B) Nav channel punctate distribution (green dots). Nav label was also found on the surface membranes of interneurons (e.g., VML cells) where their distribution is more uniform. Two interneuron cell bodies are outlined with a dashed yellow line. (C) Merged MAP2 and Nav labeling. Note that these images show the combined z-layers of the scan through the $16 \mu \mathrm{m}$ slice but that looking through the individual successive scans at varying depth (0.5 $\mu \mathrm{m}$ apart) can help determine the proximity between MAP2 and Nav labeling. (D) Nav and MAP2 labeling in a more distal portion of VML ( 200 $\mu \mathrm{m}$ from tSF boundary). (E) Enlarged view of one dendrite in the image displayed in (D) selected for quantification of Nav channels. The proximity between the labeled Nav channels and the labeled MAP2 inside dendrites allows us to confirm that the channels were in the membrane of a PC dendrite. Two puncta are indicated with yellow arrows. Note that the Nav and MAP2 labeling does not need to overlap directly since MAP2 is located inside the dendrite and Nav channels are in the membrane. We thus expect some of the puncta to be slightly separated (in the $x-y$ plane or the $z$ plane) from the MAP2 labeling. (F) Western blot analysis of brain tissue demonstrating the specificity of Anti-pan Nav antibody. The western blot of the tissue processed along with a protein ladder displayed a single band at $\sim 250-260 \mathrm{kDa}$

microtubules inside dendrites (Deng et al., 2005). By using thin brain slices (15-20 $\mu \mathrm{m})$ and high-resolution confocal scans we were able to precisely localize the labeled channels relative to PCs apical dendrites (Figures 3A-E). The pattern of expression of Nav channels in the dendrites followed a punctate pattern, as described previously (Turner et al., 1994), that is visibly different from the more uniform expression pattern in the soma of interneurons (Figure 3B) or in axons. Prior to visualizing the Nav labeling, we selected in each scan 2-4 portions of dendrites that are clearly delineated by the MAP2 labeling. We then visualized in $3 \mathrm{D}$ (moving through the $\mathrm{z}$-plane with the imaging software) the position of each punctum in the vicinity of the dendrite portion selected for quantification. Since Nav channels are in the membrane of the dendrites and MAP2 proteins are inside the dendrite, Nav puncta were immediately adjacent (in the $x-y$ plane or the z-plane) to the MAP2 labeling or overlapping.

Our dataset is based on images from 15 brain slices (five slices each from three fish). In each slice, all three segments were scanned and quantified thereby assuring that differences in immunolabeling clarity from slice to slice could not cause a bias in quantification across segments. Scans starting at the tSF layer and tiled to extend beyond $600 \mu \mathrm{m}$ into the molecular layer (Figure 4) allowed us to evaluate Nav expression as a function of segment and location along the ventral-dorsal axis (i.e., proximal-distal to the soma). We localized and counted Nav puncta on 727 portions of dendrites representing over $21,400 \mu \mathrm{m}$ of dendrites (Figure 5). We found a large dendrite-to-dendrite variability in puncta density with some long portions of dendrites containing no puncta and others being densely populated with puncta. This variability could be observed even between dendrites located side-by-side on the same scan precluding the possibility that variations in the immunolabeling process could account for this variability.

PCs can be classified in several subcategories (deep, intermediate or superficial PCs and ON-cells or OFF-cells) organized in microcolumns and their apical dendrites can overlap at the same location in the molecular layer. Our method cannot determine to which type of PC a dendrite belongs and 


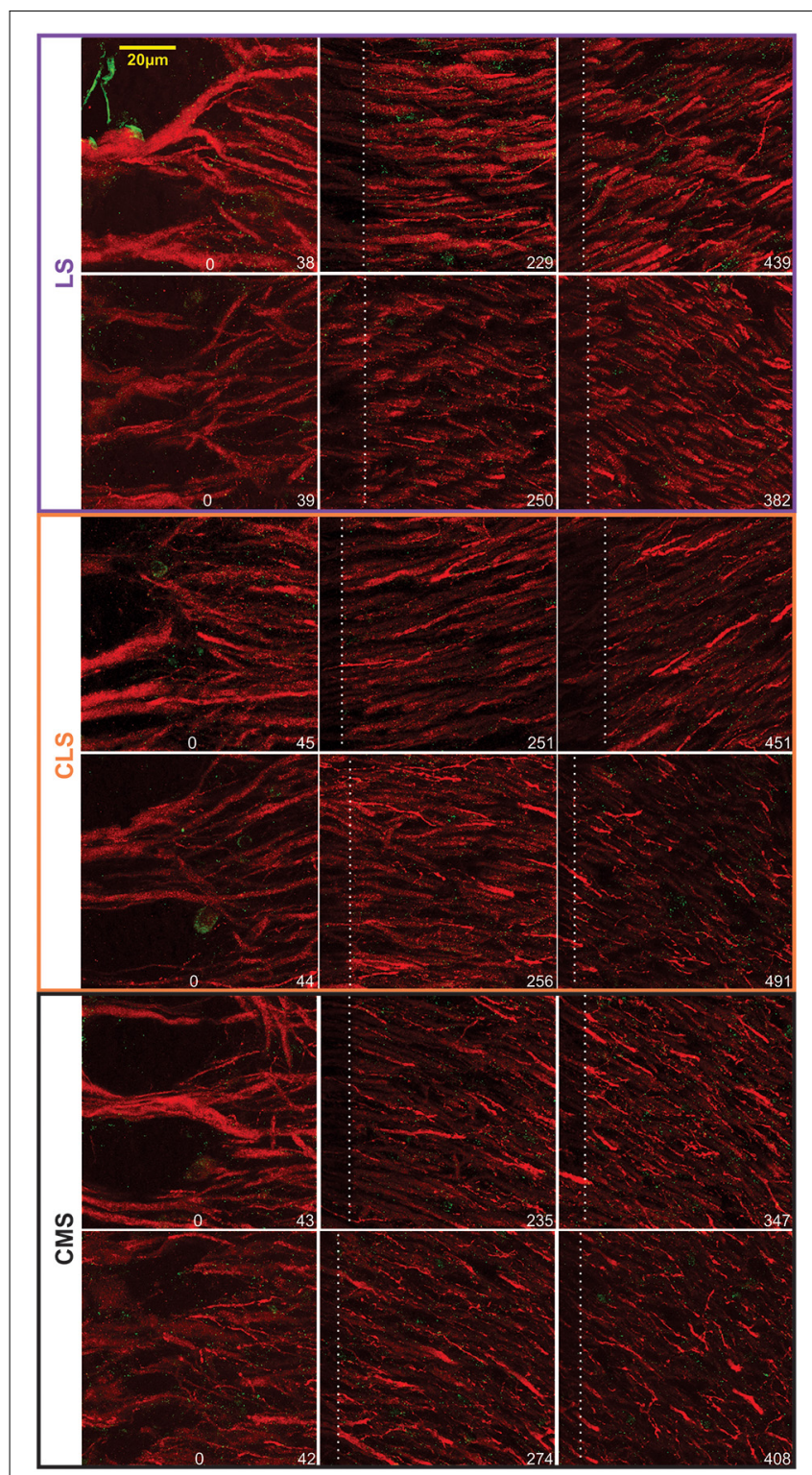

FIGURE 4 | Examples of Nav-Map 2 expression in the ELL. Confocal images from two brains (top vs. bottom rows for each map) taken at different distances from the tSF. The location of the distal edge of the image is specified in $\mu \mathrm{m}$ at the bottom right of each image. The white dashed lines indicate the edge of the previous - overlapping - scan and thus the pixels on the left of this line are bleached from the previous scan.

thus we cannot determine to what extent the variability observed is due to variations across-vs. within-subcategories.

The density of puncta per $\mu \mathrm{m}$ of dendrite was calculated for each of the $\sim 250$ dendrites per segment and we first visualize in a $3 \mathrm{D}$ histogram the proportion of dendrites with various densities as a function of dendrite position along the molecular layer (Figure 6A). The pattern of Nav expression clearly differs across segments. In LS, a large proportion of dendrites have puncta densities higher than 0.1 puncta/ $\mu \mathrm{m}$ whereas CMS dendrites have densities mostly below 0.1 puncta/ $\mu \mathrm{m}$. Our data also shows that density stays similar throughout the molecular layer up to at least $600 \mu \mathrm{m}$ away for tSF. Our data beyond $600 \mu \mathrm{m}$ are more sparse, nevertheless, the data we do have suggest that more distal dendrites express $\mathrm{Nav}$ channels with a density similar to the more proximal dendrites (see Figures 5, 6A). Our data demonstrate that dendrites in the LS have Nav puncta densities about twice as high as CMS dendrites and 1.4 times higher than CLS dendrites (Figures 6B,C). The distribution of Nav puncta along the first $600 \mu \mathrm{m}$ of dendrites (for which we have most data) was not very different across segments. Specifically, we see similar densities over the length of the dendrites in LS and CLS but channel density increased slightly in CMS for dendrites located further from tSF (Figure 6D). Therefore, our data show that, despite small differences across segments, we see a qualitatively similar distribution of Nav puncta over the entire length of dendrites considered here $(0-600 \mu \mathrm{m}$ from tSF dorsal edge; Figure 6E).

The differences in Nav channel density across segments could have a significant impact on the neuron response properties. The impact on neural dynamic and sensory processing is hard to gauge because PCs differ in many aspects between segments. Differences in ion channel composition, tuning, connectivity and more, interact intricately with the dynamics imposed by Nav channels. Even potential studies using modeling, where a single parameter can be altered (e.g., Nav channels density), presents important challenges. Indeed, all the elements of the neurons and of the network known to influence the neuron's response dynamic would need to be included to understand the effect of changing Nav density and determine if it can explain differences observed across segments. Such modeling effort is beyond the scope of this manuscript.

Nevertheless, we suggest that looking at the spontaneous activity of the neurons could give us useful insight into the effects of differences in Nav density on bursting. ISIs during spontaneous activity are largely determined by the neuron's intrinsic mechanisms since excitation is weak and relatively constant. Furthermore, burst ISIs are heavily influenced by only a few ion channels, first and foremost the Nav channels in apical dendrites. Therefore, we investigated potential differences across segments in burst ISIs during spontaneous activity. Focusing on superficial PCs, we show that burst ISIs tend to be shorter in LS than in CMS; CLS being intermediate (Figure 7; average ISIs \pm SE: $\mathrm{LS}=3.2 \pm 0.6$; CLS $=3.8 \pm 0.5$; CMS $=6.2 \pm 0.3$. Wilcoxon test: LS-CLS, $p<0.0001$; LS-CMS, $p<10^{-9}$; CLSCMS, $p<10^{-7} \cdot n=13-19$ neurons). This trend is consistent with a previous observations (Turner et al., 1996; Mehaffey et al., 2008) and could potentially be attributed in part to the higher density of Nav channels in LS (see "Discussion" section).

\section{DISCUSSION}

\section{Dendritic Nav Channel Expression and Firing Patterns}

The principal finding of the present experiment was that LS exhibits the highest dendritic Nav channel density, followed by CLS and CMS. The difference was obvious in the proximal dendritic regions of apical dendrites that support 


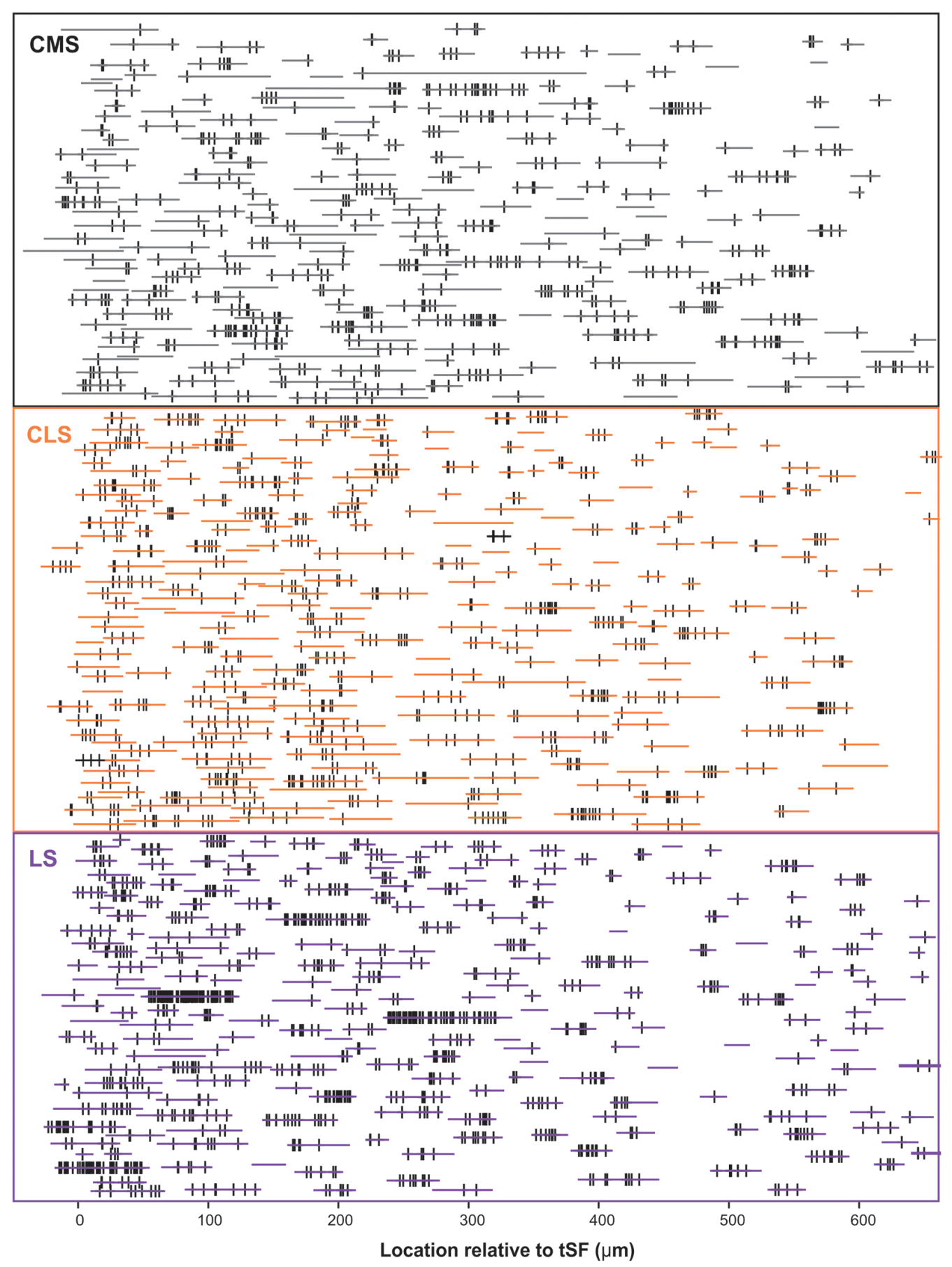

FIGURE 5 | Schematic representation of the entire dataset. The position and length of each dendrite quantified is depicted by the horizontal colored lines. The position of each Nav punctum identified is marked by a vertical black line. The vertically stacked arrangement of the dendrites loosely follows the order of processing and thus data from a given slice/brain are found in adjacent rows of the stack. We quantified 727 dendrites in 15 brain slices, totaling $>21,400 \mu \mathrm{m}$ of dendrite, and identified 2,435 puncta of Nav labeling across segments/locations.

active backpropagation. Nav channel densities remain relatively constant along the proximo-distal axes of the apical dendrites in all segments.

Previous studies have established the subcellular distribution and function of TTX sensitive Nav channels using immunocytochemistry and electrophysiology techniques (Turner et al., 1994). Punctate regions of Nav immunolabel were detected in PC somata, basal and apical dendrites. The previous EM study (Turner et al., 1994) pointed out that regions of denser labeling along a dendrite could be separated by longer portions 


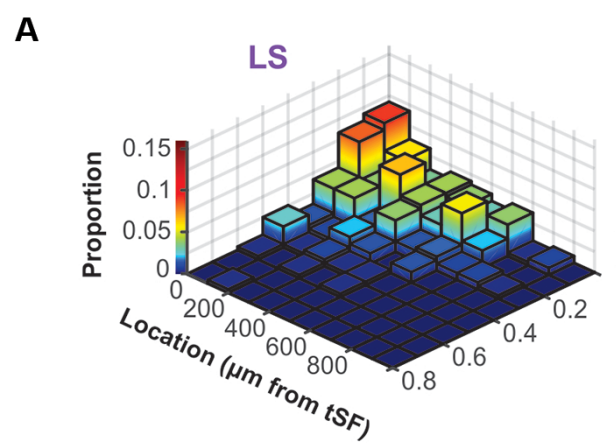

B

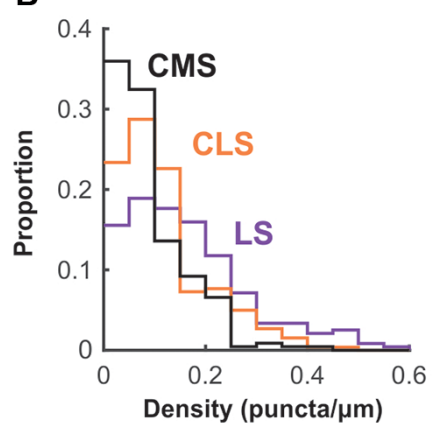

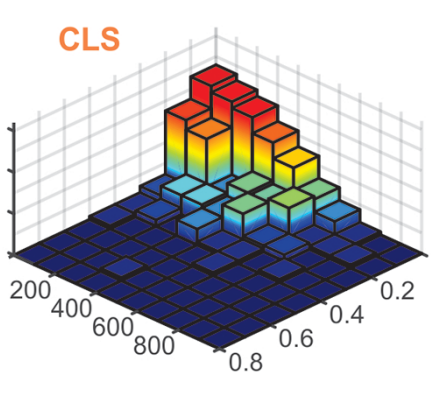

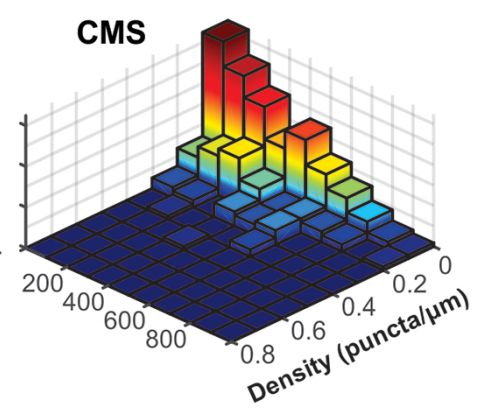

C
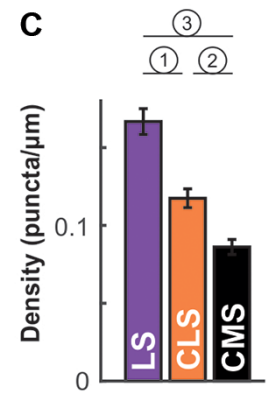

D

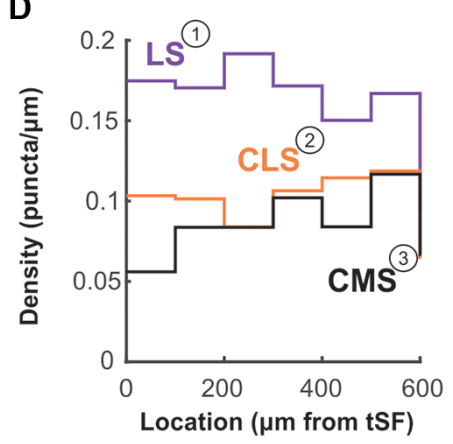

E

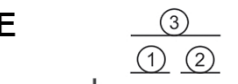

FIGURE 6 | Nav channel expression density differs across maps. (A) Histogram of channel density per dendrite as a function of location. For each dendrite (LS, $n=238$; CLS, $n=261$; CMS, $n=228$ ) channel density (puncta/ $\mu \mathrm{m}$ ) was calculated. The $z$-axis (also color-mapped) shows the proportion of dendrites in each location/density bin (i.e., sum of all bin heights is 1 in each panel). These data show that densities in CMS are largely constrained to the $0-0.1$ puncta/ $\mu \mathrm{m}$ range whereas densities are equally distributed in the 0-0.1 and 0.1-0.2 range (and higher) for LS (see panels B,C). Also, channel density seems to be equally distributed along at least the first $600 \mu \mathrm{m}$ of the molecular layer (see panels $\mathbf{D}, \mathbf{E}$ ). Note that overall height of the bins tends to decrease with increasing distance from tSF simply because fewer dendrites were quantified distally compared to proximal locations. (B) Histogram of channel density across maps. The data displayed in (A) were collapsed across all locations to clearly show the differences in puncta density across maps. LS clearly has a higher proportion of dendrites with high density ( $>0.1$ puncta/ $\mu \mathrm{m}$ ) compared with CMS and thus a lower proportion of dendrites with low density $(<0.1$ puncta/ $\mu \mathrm{m})$. See panel (C) for a listing of statistical differences. (C) Average puncta density is significantly different across segments and decreases from LS to CMS. Since the data are not normally distributed (see panel B) we tested pairwise differences using a Wilcoxon test: (1) $p=0.000004$; (2) $p=0.0002$; and (3) $p=10^{-15}$. (D) Channel density is similar across location in the molecular layer. Average channel density was calculated for dendrite positioned at various distances for tSF. Location does not influence the observed puncta density in LS and CLS [One-way ANOVA: (1) $p=0.82$; (2) $p=0.1$ ] but density increases slightly with distance in CMS [One-way ANOVA: (3) $p=0.005$ ]. The analysis shown in this panel and in panel (E) took into account dendrites located $<600 \mu \mathrm{m}$ only since our data for locations $>600 \mu \mathrm{m}$ is sparse. (E) Nav channel distribution across the molecular layer is similar for all three maps. We calculated an average channel location across the $600 \mu \mathrm{m}$ of the molecular layer considered here to compare the distribution of channels along the dendrites for the three maps. To do so, we normalized our puncta counts to account for the length of dendrites quantified in each location bin. Therefore, an average location of $300 \mu \mathrm{m}$ indicates that channels are uniformly distributed across the dendritic length considered here. This average is similar for all three maps ranging from $294 \mu \mathrm{m}$ to $323 \mu \mathrm{m}$ but the small differences are significant [Wilcoxon: (1) $p=0.0001 ;(2) p=0.003$; and (3) $\left.p=10^{-11}\right]$.

of dendrites with little labeling. Our study confirms that channel density varied widely from one portion of the dendrite to the next. Note that our study does not distinguish between dendrites belonging to ON-cell, OFF-cells or interneurons. Part of the variability observed could be due to differences across cell types If this was the case, you would expect to see two or three clusters of channels densities (one for each cell types) but we observe a mono-modal distribution instead. Future studies labeling for cell identity and for Nav could test this hypothesis.

Dendritic Nav channels allow the active propagation of an antidromic spike over around $200 \mu \mathrm{m}$ of proximal apical dendrite. The resulting DAP at the soma after each somatic spike underlies the production of burst discharge. Focal ejections of TTX inactivates Nav channels, decreasing spike frequency (Turner et al., 1994; Oswald et al., 2004). However, higher conductance from Nav channels does not necessarily lead to an increase in spike frequency. Decreased $\mathrm{Na}^{+}$conductance in dendrites can increase the excitability of the soma by delaying the DAP thereby enhancing the so-called "ping-pong" dendrosomatic dynamics that underlies burst generation (Fernandez et al., 2005). There is thus a non-monotonic relationship between $\mathrm{Na}^{+}$conductance and cell excitability (Fernandez et al., 2005) or the amount of information carried by bursts (Doiron et al., 2007) with a maximum at intermediate values. Nevertheless, modeling studies indicate that an increase in dendritic conductance from Nav channels systematically causes increased burst rates (Doiron et al., 2007).

Backpropagation has been characterized as traveling up $200 \mu \mathrm{m}$ before the active propagation is not detected. Our results, and the original study that identified the Nav channel's presence in the dendrites (Turner et al., 1994), show that the channels are distributed along the majority of the dendritic 

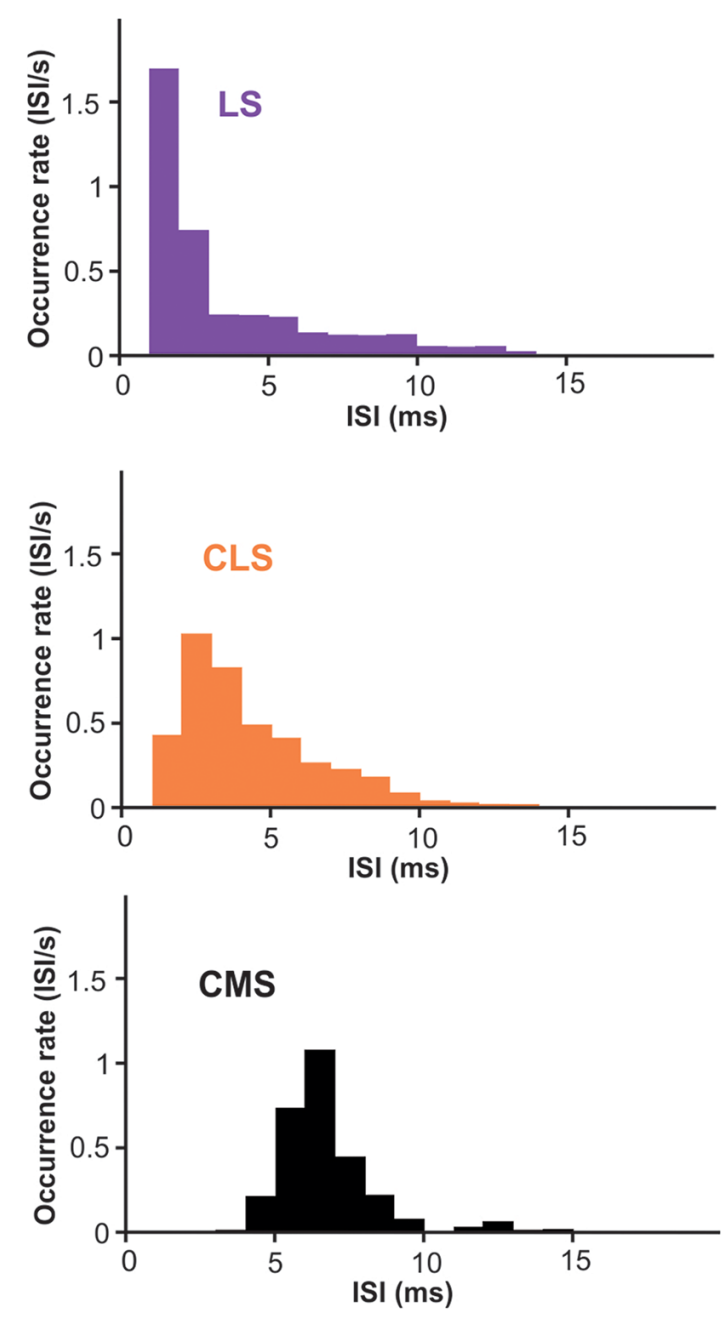

FIGURE 7 | Inter-spike intervals (ISIs) histogram for burst-spikes during spontaneous activity. Burst of spikes was identified as described in the methods and the interval between the spikes in each burst contributed to the histograms displayed. The average interval is shorter in LS than CMS with significant differences across all three segments (averages \pm SE:

$\mathrm{LS}=3.2 \pm 0.6 ; \mathrm{CLS}=3.8 \pm 0.5 ; \mathrm{CMS}=6.2 \pm 0.3$. Wilcoxon test: LS-CLS, $p<0.0001$; LS-CMS, $p<10^{-9}$; CLS-CMS, $p<10^{-7} . n=13-19$ neurons).

tree. The role of these channels, past the $200 \mu \mathrm{m}$ where backpropagation is evident, is unknown. One possibility is that they contribute to the DAP but the current they generate in the more distal dendrites is too small to be clearly identified. The DAP is indeed likely to be reduced in distal apical dendrites due to the presence of Kv3.3 channels (Deng et al., 2005) producing an after-hyperpolarization. Another possibility involves an interaction between the channels and synaptic inputs. GABAergic inputs have been shown to influence the DAP generation (Mehaffey et al., 2005), it is therefore not unlikely that the $\mathrm{Na}+$ current in the distal dendrites shapes and affects the dynamics of synaptic inputs. Given the important role of feedback inputs onto these apical dendrites the presence of the channels near these synapses could alter the neuron's response properties significantly.

\section{Segment-Specific Regulation of Bursting Mechanisms}

The effect of variations in the DAP current is particularly hard to predict because several other currents overlap with the depolarization from dendritic Nav channels. K channels, muscarinic or 5HT receptors, and GABAergic inputs can influence the after-potential (Márquez et al., 2013). In particular, somatic spikes are followed by both fast and slow AHPs that help repolarize the cell and lengthen the interval to the next spikes (Turner et al., 2002). Therefore, the DAP and the AHP have opposite influences on bursting. ELL expresses two different subtypes of SK channels (SK1: dendrites; SK2: soma) that cause AHPs (Ellis et al., 2007b, 2008) following a gradient where LS has a denser distribution than CMS. The relatively short DAP (8-10 ms) temporally overlaps with the longer AHP and their strength varies with PC subtype. It is possible that higher expression of dendritic Nav channels may partially compensate higher hyperpolarizing currents and allow these currents to be modulated with different gains.

Several mechanisms for external modulation of these hyperpolarizing and depolarizing currents have been characterized. Inhibitory interneurons are prevalent in the ELL (e.g., VML cells or granular cells) and synapse both on PC's soma and dendritic arbor (Berman and Maler, 1999). Dendritic application of $\mathrm{GABA}_{\mathrm{A}}$ agonist can affect the dendritic leak conductance leading to a DAP reduction and thus have a divisive effect on the cell's input-output relationship (Mehaffey et al., 2005). In contrast, somatic inhibition has a subtractive effect in suprathreshold regime and divisive in subthreshold regime since the reversal potential of $\mathrm{GABA}_{\mathrm{A}}$ channels is close to the neuron's resting potential (Doiron et al., 2002). Therefore, somatic inhibition could also potentially interact with the subthreshold dynamic influenced by the DAP and AHPs. Since the inhibitory surround input onto PCs varies across segments (Hofmann and Chacron, 2017) and the proportion of inhibitory interneurons (Supplementary Figure S3) also varies across segments, GABAergic inhibition is another segment-specific factor that could interact with the current from dendritic Nav channels.

5-HT is an important modulator of social behavior that is released during communication and LS shows the highest 5-HT innervation (Johnston et al., 1990). Interestingly, the innervation pattern is also layer specific across segments, where LS shows dense expression in the PCL and VML. 5-HT increases burst firing across segments with the greatest effect in the LS which is consistent with its expression density (Deemyad et al., 2011). These effects are mediated by 5-HT2 receptors (Larson et al., 2014) that increase PC excitability and bursting via downregulation of SK channel and M-type potassium channel currents that contribute to the AHP (Deemyad et al., 2013).

Given that several factors affect the shape of spike's afterpotential and the bursting dynamic, we cannot be certain that the shorter burst ISIs we observed in LS is due to the denser Nav expression but it is a plausible factor. A strong DAP, that peaks a few ms after the spike, could explain the relatively high probability of having 2-4 ms ISIs in LS whereas the 
longer-lasting AHPs strongly influencing LS ON-cells could lead to the relatively low probability of ISIs longer than $5 \mathrm{~ms}$. The longer burst ISIs observed in CMS could result from the lower Nav current since it can delay the DAP (Fernandez et al., 2005) and is not opposed by a strong AHP in superficial PCs. We, therefore, propose that differences in Nav channel expression interact with other aspects of the neuron's dynamic to influence the spiking patterns.

\section{Bursts and Neural Coding}

Bursts have a well-defined role in coding for specific temporal features of sensory signals. The relationship between patterns of spikes within a burst can further signal details about the feature that triggered the burst (Oswald et al., 2007). Specifically, the ISI within the burst is correlated with the amplitude and slope of the upstroke it signals. Studies in several sensory systems have shown that burst structure can carry information about the stimulus (Guido et al., 1995; Lesica and Stanley, 2004) and that this information is behaviorally relevant (Marsat and Pollack, 2010). Our results point to the possibility that variations in Nav channels may influence the burst dynamic to adjust the correlation between burst ISI and stimulus features. By adjusting the range of ISI coding for specific portions of stimulus space, each ELL segment could have its burst-code adjusted for slightly different stimulus features. We already know that bursts are involved in processing different signals across segments since bursts signal the presence of certain types of chirps in LS but not the other segments (Marsat et al., 2009). Also, bursts in CLS and CMS might be more specifically dedicated to signaling prey-like stimuli (Nelson and Maciver, 1999). Differences in Nav expression we describe could contribute to these different roles of burst coding across segments.

We started this article by pointing out two perspectives on neural heterogeneity. One highlights how similar neural outputs can arise from diverse combinations of neural properties. The other focuses on the need to vary neural physiology in order to adjust and specialize cells for different purposes. Our study does not determine what role the variability in dendritic Nav channel expression plays: compensating for other neural properties to keep burst coding functioning similarly across segments or on the contrary adjusting burst coding for specific roles. Since these two possibilities are not exclusive, we speculate both may be at work. The differences we describe in our study could partly compensate for other variations in PC properties while at the same time influence burst structure and coding to improve the ability of different segments to perform specific tasks.

\section{REFERENCES}

Ban, Y., Smith, B. E., and Markham, M. R. (2015). A highly polarized excitable cell separates sodium channels from sodium-activated potassium channels by more than a millimeter. J. Neurophysiol. 114, 520-530. doi: 10.1152/jn. 00475.2014

Bastian, J., Chacron, M. J., and Maler, L. (2004). Plastic and nonplastic pyramidal cells perform unique roles in a network capable of adaptive redundancy reduction. Neuron 41, 767-779. doi: 10.1016/s0896-6273(04)00071-6

\section{DATA AVAILABILITY}

All datasets generated for this study are included in the manuscript and/or the Supplementary Files.

\section{ETHICS STATEMENT}

This study was carried out in accordance with the recommendations of Animal Welfare Act, the Public Health Service Policy on Humane Care and Use of Laboratory Animals, the Guide for the Care and Use of Laboratory Animals established by the Office of Laboratory Animal Welfare. The protocol was approved by the West Virginia University Institutional Animal Care and Use Committee.

\section{AUTHOR CONTRIBUTIONS}

GM designed the research, supervised the experiments and data analysis and wrote the article. SM performed the Nav immunolabeling experiments and analyzed them and wrote the article. KA performed the GABA immunolabeling experiments and analyzed them and wrote the article. DW performed the electrophysiological recordings and analyzed them.

\section{FUNDING}

This research was supported by an National Science Foundation (NSF; IOS \#1557846) and West Virginia University Research Corporation.

\section{ACKNOWLEDGMENTS}

We thank Dr Andrew Dacks and Dr Saravanan Kolandaivelu for offering their time, resources and guidance to help with some of the experimental procedures. Dr Michael Markham and Dr Vielka Salazar and their lab members were helpful in establishing the immunolabeling protocol. We also thank Dr Maurice Chacron and Dr Len Maler for useful discussions about this project.

\section{SUPPLEMENTARY MATERIAL}

The Supplementary Material for this article can be found online at: https://www.frontiersin.org/articles/10.3389/fncir.2019.000 41/full\#supplementary-material

Bastian, J., and Courtright, J. (1991). Morphological correlates of pyramidal cell adaptation rate in the electrosensory lateral line lobe of weakly electric fish. J. Comp. Physiol. A 168, 393-407. doi: 10.1007/bf00199600

Bastian, J., and Nguyenkim, J. (2001). Dendritic modulation of burst-like firing in sensory neurons. J. Neurophysiol. 85, 10-22. doi: 10.1152/jn.2001. 85.1.10

Berman, N. J., and Maler, L. (1999). Neural architecture of the electrosensory lateral line lobe: adaptations for coincidence detection, a sensory searchlight and frequency-dependent adaptive filtering. Neuron 202, 1243-1253. 
Chacron, M. J., Longtin, A., and Maler, L. (2011). Efficient computation via sparse coding in electrosensory neural networks. Curr. Opin. Neurobiol. 21, 752-760. doi: 10.1016/j.conb.2011.05.016

Clarke, S. E., Longtin, A., and Maler, L. (2015). Contrast coding in the electrosensory system: parallels with visual computation. Nat. Rev. Neurosci. 16, 733-744. doi: 10.1038/nrn4037

Deemyad, T., Maler, L., and Chacron, M. J. (2011). Inhibition of SK and $\mathrm{M}$ channel-mediated currents by 5 -HT enables parallel processing by bursts and isolated spikes. J. Neurophysiol. 105, 1276-1294. doi: 10.1152/jn. 00792.2010

Deemyad, T., Metzen, M. G., Pan, Y., and Chacron, M. J. (2013). Serotonin selectively enhances perception and sensory neural responses to stimuli generated by same-sex conspecifics. Proc. Natl. Acad. Sci. U S A 110, 19609-19614. doi: 10.1073/pnas.1314008110

Deng, Q., Rashid, A. J., Fernandez, F. R., Turner, R. W., Maler, L., and Dunn, R. J. (2005). A C-terminal domain directs Kv3.3 channels to dendrites. J. Neurosci. 25, 11531-11541. doi: 10.1523/JNEUROSCI. 3672-05.2005

Doiron, B., Laing, C., Longtin, A., and Maler, L. (2002). Ghostbursting: a novel neuronal burst mechanism. J. Comput. Neurosci. 12, 5-25. doi: 10.1023/A:1014921628797

Doiron, B., Oswald, A.-M. M., and Maler, L. (2007). Interval coding. II. Dendritedependent mechanisms. J. Neurophysiol. 97, 2744-2757. doi: 10.1152/jn. 00988.2006

Duménieu, M., Oulé, M., Kreutz, M. R., and Lopez-Rojas, J. (2017). The segregated expression of voltage-gated potassium and sodium channels in neuronal membranes: functional implications and regulatory mechanisms. Front. Cell. Neurosci. 11:115. doi: 10.3389/fncel.2017.00115

Ellis, L. D., Krahe, R., Bourque, C. W., Dunn, R. J., and Chacron, M. J. (2007a). Muscarinic receptors control frequency tuning through the downregulation of an a-type potassium current. J. Neurophysiol. 98, 1526-1537. doi: 10.1152/jn. 00564.2007

Ellis, L. D., Mehaffey, W. H., Harvey-Girard, E., Turner, R. W., Maler, L., and Dunn, R. J. (2007b). SK channels provide a novel mechanism for the control of frequency tuning in electrosensory neurons. J. Neurosci. 27, 9491-9502. doi: 10.1523/JNEUROSCI.1106-07.2007

Ellis, L. D., Maler, L., and Dunn, R. J. (2008). Differential distribution of SK channel subtypes in the brain of the weakly electric fish Apteronotus leptorhynchus. J. Comp. Neurol. 507, 1964-1978. doi: 10.1002/cne. 21597

Fernandez, F. R., Mehaffey, W. H., and Turner, R. W. (2005). Dendritic $\mathrm{Na}^{+}$ current inactivation can increase cell excitability by delaying a somatic depolarizing afterpotential. J. Neurophysiol. 94, 3836-3848. doi: 10.1152/jn. 00653.2005

Gabbiani, F., Metzner, W., Wessel, R., and Koch, C. (1996). From stimulus encoding to feature extraction in weakly electric fish. Nature 384, 564-567. doi: $10.1038 / 384564 \mathrm{a} 0$

Guido, W., Lu, S. M., Vaughan, J. W., Godwin, D. W., and Sherman, S. M. (1995). Receiver operating characteristic (ROC) analysis of neurons in the cat's lateral geniculate nucleus during tonic and burst response mode. Vis. Neurosci. 12, 723-741. doi: 10.1017/s0952523800008993

Hille, B. (2001). Ion Channels of Excitable Membranes. 3rd Edn. Sunderland, Massachusetts, USA: Sinauer Associates.

Hofmann, V., and Chacron, M. J. (2017). Differential receptive field organizations give rise to nearly identical neural correlations across three parallel sensory maps in weakly electric fish. PLoS Comput. Biol. 13:e1005716. doi: 10.1371/journal.pcbi.1005716

Johnston, S. A., Maler, L., and Tinner, B. (1990). The distribution of serotonin in the brain of Apteronotus leptorhynchus: an immunohistochemical study. J. Chem. Neuroanat. 3, 429-465.

Kawasaki, M. (2005). "Physiology of tuberous electrosensory systems," in Electroreception, eds T. H. Bullock, C. D. Hopkins, A. N. Popper and R. R. Fay (New York, NY: Springer), 154-194.

Kepecs, A., Wang, X.-J., and Lisman, J. (2002). Bursting neurons signal input slope. J. Neurosci. 22, 9053-9062. doi: 10.1523/JNEUROSCI.22-20-09053.2002

Krahe, R., Bastian, J., and Chacron, M. J. (2008). Temporal processing across multiple topographic maps in the electrosensory system. J. Neurophysiol. 100, 852-867. doi: 10.1152/jn.90300.2008
Krahe, R., and Gabbiani, F. (2004). Burst firing in sensory systems. Nat. Rev. Neurosci. 5, 13-23. doi: 10.1038/nrn1296

Krahe, R., and Maler, L. (2014). Neural maps in the electrosensory system of weakly electric fish. Curr. Opin. Neurobiol. 24, 13-21. doi: 10.1016/j.conb.2013. 08.013

Larson, E. A., Metzen, M. G., and Chacron, M. J. (2014). Serotonin modulates electrosensory processing and behavior via 5-HT2-like receptors. Neuroscience 271, 108-118. doi: 10.1016/j.neuroscience.2014.04.033

Lesica, N. A., and Stanley, G. B. (2004). Encoding of natural scene movies by tonic and burst spikes in the lateral geniculate nucleus. J. Neurosci. 24, 10731-10740. doi: 10.1523/JNEUROSCI.3059-04.2004

Lizbinski, K. M., Marsat, G., and Dacks, A. M. (2018). Systematic analysis of transmitter coexpression reveals organizing principles of local interneuron heterogeneity. eNeuro 5:ENEURO.0212-18.2018. doi: 10.1523/eneuro.0212 $-18.2018$

Maler, L. (1979). The posterior lateral line lobe of certain gymnotoid fish: quantitative light microscopy. J. Comp. Neurol. 183, 323-363. doi: 10.1002/cne. 901830208

Maler, L. (2007). Neural strategies for optimal processing of sensory signals. Prog. Brain Res. 165, 135-154. doi: 10.1016/S0079-6123(06)65009-7

Maler, L. (2009). Receptive field organization across multiple electrosensory maps. I. Columnar organization and estimation of receptive field size. J. Comp. Neurol. 516, 376-393. doi: 10.1002/cne.22124

Márquez, B. T., Krahe, R., and Chacron, M. J. (2013). Neuromodulation of early electrosensory processing in gymnotiform weakly electric fish. J. Exp. Biol. 216, 2442-2450. doi: 10.1242/jeb.082370

Marsat, G., and Pollack, G. S. (2006). A behavioral role for feature detection by sensory bursts. J. Neurosci. 26, 10542-10547. doi: 10.1523/JNEUROSCI.222106.2006

Marsat, G., and Pollack, G. S. (2010). The structure and size of sensory bursts encode stimulus information but only size affects behavior. J. Comp. Physiol. A 196, 315-320. doi: 10.1007/s00359-010-0514-8

Marsat, G., and Pollack, G. S. (2012). Bursting neurons and ultrasound avoidance in crickets. Front. Neurosci. 6:95. doi: 10.3389/fnins.2012.00095

Marsat, G., Proville, R. D., and Maler, L. (2009). Transient signals trigger synchronous bursts in an identified population of neurons. J. Neurophysiol. 102, 714-723. doi: 10.1152/jn.91366.2008

Mehaffey, W. H., Doiron, B., Maler, L., and Turner, R. W. (2005). Deterministic multiplicative gain control with active dendrites. J. Neurosci. 25, 9968-9977. doi: 10.1523/JNEUROSCI.2682-05.2005

Mehaffey, W. H., Maler, L., and Turner, R. W. (2008). Intrinsic frequency tuning in ELL pyramidal cells varies across electrosensory maps. J. Neurophysiol. 99, 2641-2655. doi: 10.1152/jn.00028.2008

Metzner, W., and Juranek, J. (1997). A sensory brain map for each behavior? Proc. Natl. Acad. Sci. U S A 94, 14798-14803. doi: 10.1073/pnas.94.26. 14798

Metzner, W., Koch, C., Wessel, R., and Gabbiani, F. (1998). Feature extraction by burst-like spike patterns in multiple sensory maps. J. Neurosci. 18, 2283-2300. doi: 10.1523/JNEUROSCI.18-06-02283.1998

Nelson, M. E., and Maciver, M. A. (1999). Prey capture in the weakly electric fish Apteronotus albifrons: sensory acquisition strategies and electrosensory consequences. J. Exp. Biol. 202, 1195-1203.

Olesen, O. F. (1994). Expression of low molecular weight isoforms of microtubuleassociated protein 2. Phosphorylation and induction of microtubule assembly in vitro. J. Biol. Chem. 269, 32904-32908.

Oswald, A.-M. M., Chacron, M. J., Doiron, B., Bastian, J., and Maler, L. (2004). Parallel processing of sensory input by bursts and isolated spikes. J. Neurosci. 24, 4351-4362. doi: 10.1523/JNEUROSCI.0459-04.2004

Oswald, A.-M. M., Doiron, B., and Maler, L. (2007). Interval coding. I. Burst interspike intervals as indicators of stimulus intensity. J. Neurophysiol. 97, 2731-2743. doi: 10.1152/jn.00987.2006

Prešern, J., Triblehorn, J. D., and Schul, J. (2015). Dynamic dendritic compartmentalization underlies stimulus-specific adaptation in an insect neuron. J. Neurophysiol. 113, 3787-3797. doi: 10.1152/jn. 00945.2014

Prinz, A. A., Bucher, D., and Marder, E. (2004). Similar network activity from disparate circuit parameters. Nat. Neurosci. 7, 1345-1352. doi: 10.1038/ nn1352 
Turner, R. W., Lemon, N., Doiron, B., Rashid, A. J., Morales, E., Longtin, A., et al. (2002). Oscillatory burst discharge generated through conditional backpropagation of dendritic spikes. J. Physiol. 96, 517-530. doi: 10.1016/s0928-4257(03)00007-x

Turner, R., Maler, L., Deerinck, T., Levinson, S., and Ellisman, M. (1994). TTX-sensitive dendritic sodium channels underlie oscillatory discharge in a vertebrate sensory neuron. J. Neurosci. 14, 6453-6471. doi: 10.1523/JNEUROSCI.14-11-06453.1994

Turner, R. W., Plant, J. R., and Maler, L. (1996). Oscillatory and burst discharge across electrosensory topographic maps. J. Neurophysiol. 76, 2364-2382. doi: 10.1152/jn.1996.76.4.2364
Conflict of Interest Statement: The authors declare that the research was conducted in the absence of any commercial or financial relationships that could be construed as a potential conflict of interest.

Copyright (C) 2019 Motipally, Allen, Williamson and Marsat. This is an open-access article distributed under the terms of the Creative Commons Attribution License (CC BY). The use, distribution or reproduction in other forums is permitted, provided the original author(s) and the copyright owner(s) are credited and that the original publication in this journal is cited, in accordance with accepted academic practice. No use, distribution or reproduction is permitted which does not comply with these terms. 